\title{
Guiding the Search in the NO Region of the Phase Transition Problem with a Partial Subsumption Test
}

\author{
Samuel Wieczorek ${ }^{1, *}$, Gilles Bisson ${ }^{2}$, and Mirta B. Gordon ${ }^{2}$ \\ ${ }^{1}$ Laboratoire Biologie, Informatique, Mathématiques, CEA-DSV-DRDC \\ 17, avenue des martyrs, 38054 Grenoble Cedex 9, France \\ samuel.wieczorek@cea.fr \\ ${ }^{2}$ Laboratoire Leibniz-IMAG, UMR 5522 \\ 46, Avenue Félix Viallet, 38031 Grenoble Cedex, France \\ \{gilles.bisson, mirta.gordon\}@imag.fr
}

\begin{abstract}
We introduce a test, named $\pi$-subsumption, which computes partial subsumptions between a hypothesis $h$ and an example $e$, as well as a measure, the subsumption index, which quantifies the covering degree between $h$ and $e$. The behavior of this measure is studied on the phase transition problem.
\end{abstract}

Keywords: Inductive Logic Programming, similarity index, $\theta$-subsumption, partial subsumption, CSP, phase transition.

\section{Introduction}

In the learning systems using a first order logic representation, the covering test is a critical operation. It allows to check whether a given hypothesis $h$ is consistent with a given example $e$. In ILP [15], the standard covering test is the $\theta$-subsumption [17] which returns a boolean value indicating if the hypothesis covers an example or not. In such context, [4], [6] have shown that there is a phase transition, associated to the use of the $\theta$-subsumption. These works also have shown the existence of failure regions where the learning algorithms are unable to find the actual concepts. These failures occur both around the phase transition (PT) region (i.e. the mushy region between the YES and NO phases) and in some parts of the NO region.

In this paper, we extend the classical covering test by introducing the notion of partial covering between a hypothesis and an example. Our covering test does not indicate whether a hypothesis covers an example by a boolean value, but assesses the importance of the partial covering by means of a "subsumption degree" index. The evaluation of our index is done in the same framework as in [4]. We show that it is possible to create a gradient in the NO region which smoothes the abrupt jump of the PT due to the $\theta$-subsumption test. We expect such gradient will allow to efficiently guide the learning algorithms when they need to explore the NO region, for instance to be able to learn some complex conjunctive concepts.

${ }^{*}$ This work is done in the context of a $\mathrm{PhD}$ thesis in the laboratoire Leibniz. 
The paper is organized as follows. Section 2 provides some definitions and gives a brief review of the solutions to compute partial subsumptions. In Section 3 we present a heuristic to compute a partial covering and the associated subsumption degree. In Section 4 the properties of this measure are studied in the phase transition problem.

\section{Substitution and Generalization in ILP}

We restrict here to the Datalog language ${ }^{1}$ where the expressions are conjunctions of literals, each literal is built on a $n$-ary predicate which defines a property or relation between its arguments (variables or constants). First, let's give some definitions:

Definition 1. A substitution $\theta$ is a finite list of bindings $X_{i} / t_{i}$, where $X_{i}$ is a variable in $h$ and $t_{i}$ a term in $e$. The application $h \theta$ of a substitution $\theta$ to a clause $h$ is obtained by replacing all the occurrences of each variable $X_{i}$ in $h$ by the corresponding term $t_{i}$ in $e$.

Definition 2. A clause $h \theta$-subsumes a clause $e$ (denoted $h \leq e$ ) iff there exists a substitution $\theta$ such that $h \theta \subseteq e$.

Definition 3. Given two clauses $h$ and $e$ whose sets of variables are distinct, one says that $h \pi$-subsumes $e$ ( $\pi$ stands for partial) iff there exists a sub-expression $h^{\prime}$ in $h$ ( $h^{\prime} \subseteq$ $h$ ) and a substitution $\theta$ of the variables in $h^{\prime}$ such that $h^{\prime} \theta \subseteq e$.

Definition 4. Given two clauses $h$ and $e$ such that $h \leq e$, the subsumption index (denoted $\left.\mathrm{I}_{\pi}\right)$ is a function from $(h, e)$ on the interval $[0,1]$ that quantifies to which extent $h$ partially subsumes $e$, or in other terms the covering degree of $h$ ' relatively to $h$, with $h^{\prime} \subseteq h$. The index satisfies the following condition: if $h^{\prime}=h$ then $h \leq e$ and $\mathrm{I}_{\pi}=1$; when no sub-expression $h^{\prime} \leq e$, then $h$ and $e$ are completely dissimilar and $\mathrm{I}_{\pi}=0$. A computational definition of $\mathrm{I}_{\pi}$ is given in section 3.2.

To search for a partial subsumption, a solution consists in generalizing the clauses $h$ and $e$, in order to build the sub-expression $h$ '. In ILP, the most studied generalization method is the $\operatorname{lgg}$ (Least General Generalization), defined by Plotkin [17], which builds the most specific generalization that $\theta$-subsumes $h$ and $e$. Once the $\lg g$ has been built, it becomes possible to evaluate the covering degree between $h$ and $e$ by calculating the ratio between the size of the $\lg g$ and the size of $h$. Unfortunately, as the lgg contains a large number of redundant literals, a reduction step is needed to obtain the minimal form $h$ ' relevant for evaluation of the size. This reduction can be carried out by using a complete algorithms such that [7], [12] or [1], which allow to search exhaustively for a minimal solution. However, the number of possible solutions may be potentially large since it corresponds to the size of the generalization lattice. In the NO region, this problem becomes crucial since the size of $\lg g(h, e)$ is huge. To perform the reduction, some authors [5], [8] propose heuristics whose the complexity is still high: $\mathrm{O}\left(n^{4}\right)$ where $n$, according to the method, is either the number of variables or the number of literals.

1 This restriction is done for the sake of simplicity. In FOL, the functional terms can be managed by using the "flattening techniques" turning functional terms into new predicates. 


\section{Partial Subsumption and Subsumption Index}

In this section, we propose a heuristic that searches in polynomial time the substitutions $\theta$ from which the sub-expression $h^{\prime}$ can be deduced. In the rest of the paper we consider, without any loss of generality ${ }^{2}$, that the arity of the predicates of $h$ and $e$ equals 2. We use a variant of the method proposed in [2], [3] to compute a degree of subsumption between any two clauses. This method is based on two steps:

- a comparison step to evaluate a local degree of subsumption between all pairs of variables in $h$ and $e$. These values are stored in a subsumption matrix SUB;

- a matching step between the variables of $h$ and $e$, guided by the content of SUB. This second step computes the substitutions $\theta$ and the subsumption index.

\subsection{Local Comparison Between Variables}

The work [2] allows to compute a similarity index between any two clauses $h$ and $e$. For a given pair of variables $x_{i}$ in $h$ and $a_{m}$ in $e$, the main idea is to consider that their similarity is larger when they appear in common predicates at the same position, and it increases further if their neighbouring variables (i.e. the variables occurring in the same literals as $x_{i}$ and $a_{m}$ ) are themselves similar to each other. This recursive definition allows to formulate the calculus under the form of a system of equations and to ensure that the computed values reflect the similarity between the relational structures of $h$ and $e$. We have enhanced the original work in several keypoints, the main modification was to replace the notion of similarity between $x_{i}$ and $a_{m}$ by the notion of degree of subsumption. Thus, we have a referent (the hypothesis $h$ ) and a target (the example $e$ ) and the index is no more symmetrical. Here is the algorithm:

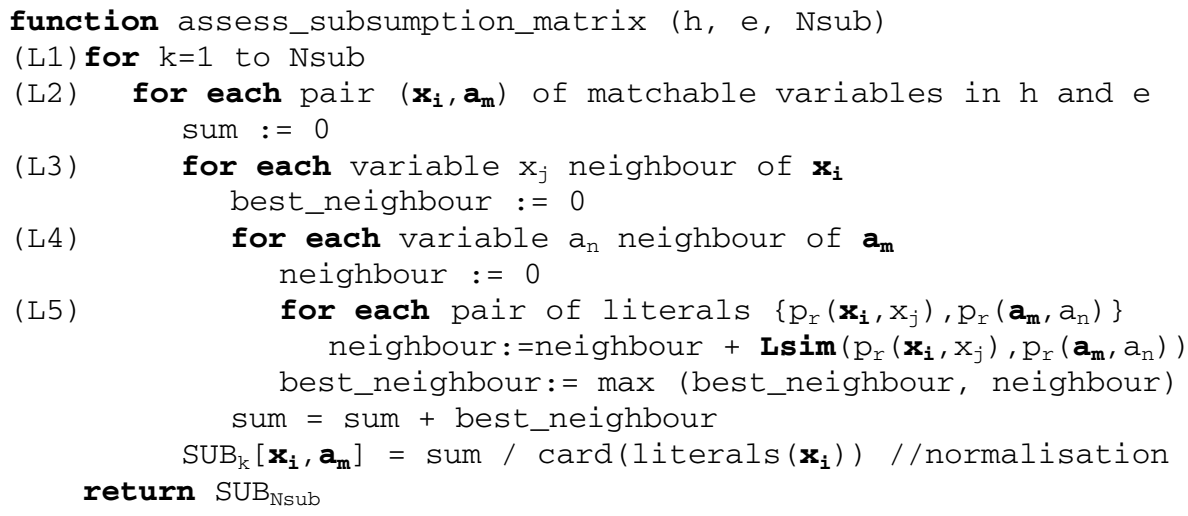

Initially all the elements of $\mathrm{SUB}_{0}$ are initialized to 1 . The loop L1 evaluates the matrix SUB through an iterative Jacobi's method since the system of equations used to estimate the degree of subsomption between variables is not linear. At each iteration, for each pair of variables $\left(x_{i}, a_{m}\right)$, we look for the best matching (i.e. the one maximizing the degree of subsumption) of the neighbours $\left\{x_{1} \ldots x_{j}\right\}$ of $x_{i}$ with the

${ }^{2}$ This restriction is done to simplify the description of the algorithms. In practice, the results presented here hold for predicates of any arity (including the complexity evaluation in 3.3). 
neighbours $\left\{a_{1} \ldots a_{n}\right\}$ of $a_{m}$. The central step of the procedure is the comparison of the pairs of matchable literals $\left\{p_{r}\left(x_{i}, x_{j}\right), p_{r}\left(a_{m}, a_{n}\right)\right\}$ (i.e. which share the same predicate symbol $p_{r}$ ) through the function Lsim (see [2] for further details):

$$
\operatorname{Lsim}\left(p_{r}\left(x_{i}, x_{j}\right), p_{r}\left(a_{m}, a_{n}\right)\right)=\left(1+S U B_{k-1}\left[x_{j}, a_{n}\right]\right) / 2
$$

This function expresses the fact that the subsumption between two literals is the average of the subsumption degree between their arguments. The subsumption value between $x_{i}$ and $a_{m}$ equals 1 since they appear in the same predicate and position. For $x_{j}$ and $a_{n}$ we use the subsumption value evaluated at the previous iteration (i.e. the value of $\left.\mathrm{SUB}_{\mathrm{k}-1}\left[x_{j}, a_{n}\right]\right)$; conversely, the evaluation of $\mathrm{SUB}_{\mathrm{k}}\left[x_{j}, a_{n}\right]$ for the same literals will recursively involve the value of $\mathrm{SUB}_{\mathrm{k}-1}\left[x_{i}, a_{m}\right]$. The algorithm iterates $N_{s u b}$ times, each iteration $k$ taking implicitly into account the of $k$-th neigbours of the current variable $x_{i}$ and $a_{m}$. However, since the influence of the neighborhood geometrically decreases according to the length of the path between the variables, the values of SUB converge after a small number iterations (we use $\mathrm{N}_{\text {sub }}=5$ in our experiments).

\subsection{Global Matching of Variables}

The second step of the method looks for an optimal (partial) matching (i.e. maximizing the subsumption index) between the variables in the hypothesis and in the example. As the $\theta$-subsumption test is equivalent to a Constraint Satisfaction Problem (CSP) [6], [16], [13] we use the Minimizing Conflicts heuristic [14], which is based on a local search algorithm. The method is initialized by assigning a value to every variable and, then iteratively changes the assignment of the variables one by one in order to minimize the number of conflicts $^{3}$. It stops either when a complete assignment $\left(I_{\pi}=1\right)$ has been found or when the maximum number of iterations $N_{\max }$ has been reached and then returns the best assignment found. Two variants have been implemented: MCA_nonGuided (the assignments are not guided by SUB) and MCA_Guided (the assignments are guided by the values of SUB). We will discuss in section 4.2, the optimal value for the parameter $\mathrm{N}_{\max }$. For a given substitution $\theta$, the subsumption index $\mathrm{I}_{\pi} \in[0,1]$, corresponding to a matching of $h$ and $e$, is proportional to the sum of the degree of subsumption of the matched variables:

$$
I_{\pi}(h, e)=\left[\sum_{i}^{V_{\text {matched }}} S U B_{N \text { sub }}\left[x_{i}, \theta\left(x_{i}\right)\right] \times\left|\operatorname{literal}\left(x_{i}\right)\right|\right] / \sum_{k}^{V}\left|\operatorname{literals}\left(x_{k}\right)\right|
$$

where:

- $\mathrm{V}$ (resp. $\mathrm{V}_{\text {matched }}$ ) is the set of (resp. matched) variables in the hypothesis $h$;

- $\mathrm{SUB}_{\mathrm{Nsub}}$ denotes the matrix SUB at the last iteration;

- $\theta\left(x_{i}\right)$ is the variable in $e$ matched with the variable $x_{i}$ in $h$ by the substitution $\theta$,

- Iliterals $\left(x_{i}\right)$ is the cardinal of the set of literals in which the variable $x_{i}$ appears.

In the numerator, we weight the value of $\operatorname{SUB}_{\mathrm{Nsub}}\left[x_{i}, \theta\left(x_{i}\right)\right]$ by the number of literals of $x_{i}$. The denominator yields the index $\mathrm{I}_{\pi}$ non-symmetrical and normalized in $[0,1]$.

\footnotetext{
${ }^{3}$ Given $\theta$, a conflict occurs if there exists a literal $\mathrm{p}_{\mathrm{r}}(\mathrm{x}, \mathrm{y})$ in $h$ but no literal $\mathrm{p}_{\mathrm{r}}(\theta(\mathrm{x}), \theta(\mathrm{y}))$ in $e$.
} 


\subsection{Computational Complexity}

The computational complexity of $\mathrm{I}_{\pi}$ is the sum of the complexities of the two steps needed for its evaluation: the evaluation of the matrix SUB and the search of the optimal match between the variables. Let's consider the following parameters:

- $V_{h}$ and $V_{e}$ : average number of variables in $h$ and $e$;

- $L_{h}$ and $L_{e}$ : average number of literals in $h$ and $e$;

- $N_{\text {sub }}$ : iteration number for the computation of the matrix SUB;

- $N_{\max }$ : maximum number of iterations in the MCA algorithm.

The elementary step in the SUB calculus, is the call to the function Lsim. The algorithm is composed of a loop of $N_{s u b}$ iterations with $V_{h} \times V_{e}$ pairs of variables to assess. Each of these pairs needs to compute the subsumption between all couples of neighbouring variables. This number is of the order of $2 \times\left(L_{h} / V_{h}\right)$ for the hypothesis and $2 \times\left(L_{e} / V_{e}\right)$ for the examples, thus the number of comparisons is $4 \times\left(L_{h} \times L_{e} / V_{h} \times V_{e}\right)$ for each pair. The complexity of this step is $\mathrm{O}\left(N_{s u b} \times L_{h} \times L_{e}\right)$, with $N_{\text {sub }}$ being small (cf. 3.1). For the matching step, we consider the complexity of the algorithm MCA, composed of a main loop of $N_{\max }$ iterations. At each iteration, two functions are called, each one compares the sets of literals in $h$ and $e$, thus giving a complexity of $\mathrm{O}\left(2 \times L_{h} \times L_{e}\right)$. The average complexity of this step is $\mathrm{O}\left(N_{\max } \times L_{h} \times L_{e}\right)$. Thus, the overall computational complexity of $\mathrm{I}_{\pi}$ index is $\mathrm{O}\left(\left(N_{\text {sub }}+N_{\max }\right) \times L_{h} \times L_{e}\right)$.

\section{Study of the Index Behavior}

It has been shown [4], [6], [9] that upon the search of a substitution $\theta$ in the $\theta$-subsumption test, a phenomenon named phase transition exists for some critical values of the order parameters, namely: the constraint density in the hypothesis and the constraint tightness in the example. Given two random formulas $h$ and $e$, this transition separates [10], in the plane whose axes are the order parameters, a phase (called YES region) where $h$ has a probability close to 1 to cover $e$ and a phase (called NO region) where the solutions are sparse and the coverage probability is close to 0 . In addition, the PT region concentrates the most complex problems and correspondingly there is a peak in computational complexity [18].

\subsection{Study of the Phase Transition}

In order to compare the behavior of the partial subsumption $\mathrm{I}_{\pi}$ with the $\theta$-subsumption in the context of the PT, we use the experimental protocol defined by [4]. This protocol is based on a set random problems, each one being a pair $(h, e)$ defined by a quadruplet $(n, m, L, N)$ where the hypothesis $h$ contains $n$ variables and $m$ literals (built on $m$ different binary predicate symbols) and the example $e$ contains $L$ constants and $N$ literals for each of the $m$ predicate symbols. As in [4], we explore points in the $\langle m, L>$ plane, for values of $m \in[10,50], L \in[10,50], n=6$ and $N=100$. For each pair $(m, L)$, we generate 100 random problems and compute the subsumption index $\mathrm{I}_{\pi}$. The averages over these problems are reported on figure 1 . 
As expected, in the YES region we observe a flat zone with $\mathrm{I}_{\pi}=1$ consistent with the fact that the problems have a solution with probability 1 . In addition, the index $\mathrm{I}_{\pi}$ regularly decreases on penetrating in the NO region where the algorithm identifies partial solutions $h$ '. The value of $\mathrm{I}_{\pi}$ is proportional to the degree of subsumption between $h$ and $e$. However, the decrease of $\mathrm{I}_{\pi}$ is not linear showing that the behavior of our comparison method is different from the one of a classical Levenstein distance.

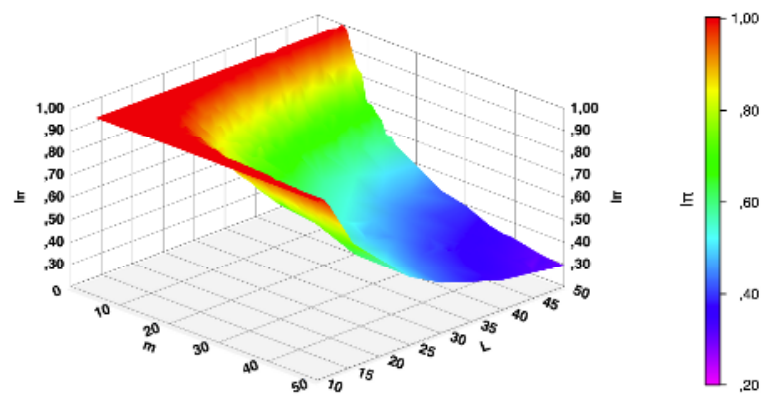

Fig. 1. Average values of index $\mathrm{I}_{\pi}$ on $<m, L>$ plane with MCA_Guided ( $N_{\max }=100$ iterations $)$

\subsection{Completeness of the Approach}

Since the proposed method is based on a heuristic, it does not guarantee that an optimal solution to the problem will always be found (i.e. a complete substitution of variables in the YES region and the largest partial solutions in the NO region). Moreover, we need to evaluate the sensibility of our method with respect to the parameter $\mathrm{N}_{\max }$ which controls the extension of search space explored to find the substitution. To answer both questions, we have modified the previous experimental protocol in such a way that, for each random problem $(h, e)$, the example $e$ is always $\theta$-subsumed by $h$ ( $h$ is named a hidden solution). In this test, as the subsumption index $\mathrm{I}_{\pi}$ should be equal to 1 for all problems, we can evaluate the minimal value of $\mathrm{N}_{\max }$ that is needed to retrieve this solution. The results are summarized in figure 2.
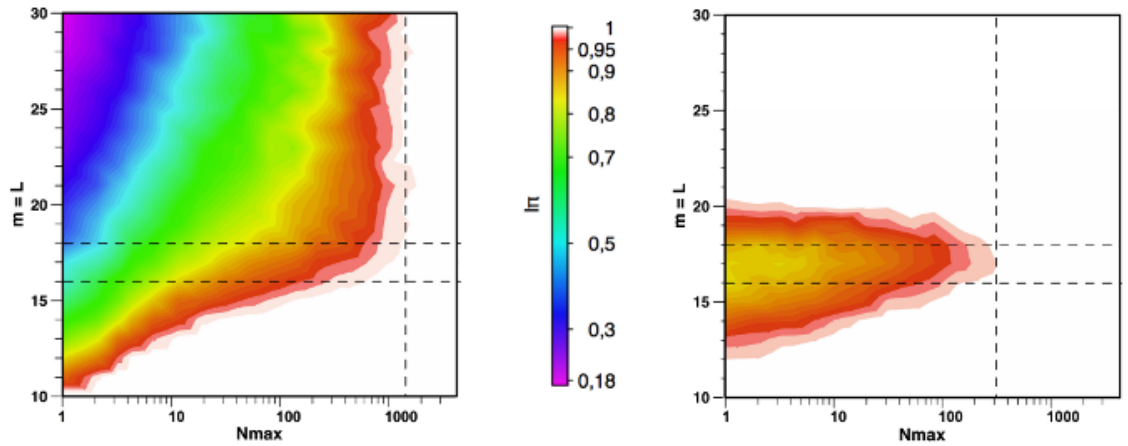

Fig. 2. Average value of index $I_{\pi}$ according to the number of iterations $N_{\max }$ for the problems along the line $m=L$; the horizontal axis has a logarithmic scale. The behavior of the algorithms MCA_nonGuided is shown on the left (figure 2a) and MCA_Guided on the right (figure 2b). 
In the YES region $(m=L \leq 16)$, both algorithms are able to find a solution with a low number of iterations (usually $\mathrm{N}_{\max }<100$ ) and when $m=L \leq 12$, the MCA_Guided finds a solution from the start with the information contained in SUB.

In the PT region $(16 \leq m=L \leq 18)$, we also observe a peak in computational complexity as described in [18], however the guided version is clearly better than the unguided one: not only it succeeds in retrieving a complete solution with a lower number of iterations $\left(\mathrm{N}_{\max } \approx 200\right.$ versus $\left.\mathrm{N}_{\max } \approx 1000\right)$ but, for a given $\mathrm{N}_{\max }$, the found solution is better than the one returned by MCA_nonGuided. A closer examination of the matrix $\mathrm{SUB}_{\mathrm{Nsub}}$ reveals that several subsumption values between the variables in $h$ and $e$ equal 1. That means that our method is unable to distinguish "from the local point of view" the different pairs of variables. Thus, it should explore more thoroughly the search space to find a satisfying solution in the matching step.

Finally, in the NO region $(m=L \geq 18)$, the two algorithms have a very different behavior. The MCA_nonGuided algorithm needs many iterations $\left(\mathrm{N}_{\max }>1000\right)$ to retrieve the solution. On the contrary, MCA_Guided finds the solution faster and for $m=L \geq 20$, the solution is found from the initial state $\left(\mathrm{N}_{\max }=0\right)$ generated using

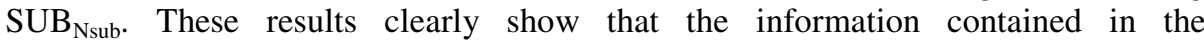
subsumption matrix SUB improves the exploration of the search space in the PT region and particularly in the NO region. For the latter, the method seems really efficient since the overall complexity just depends on the product of the size, in terms of literals in $h$ and in $e$ : $\mathrm{O}(|h| x|e|)$. This complexity is arguably lower than the one obtained by computing a $\lg g$ between $h$ and $e$ and doing the subsequent reduction.

\section{Conclusion}

In this paper, we have introduced the notion of partial subsumption (named $\pi$ subsumption) which, coupled with a subsumption index $I_{\pi}$, builds and quantifies the covering between two clauses $h$ and $e$. We have studied the behavior of the covering test in the different regions of the phase diagram on random problems.

The subsumption index suppresses the abrupt jump of the $\theta$-subsumption at the phase transition. This result is interesting since several works in ILP have shown that widely used search criteria do not allow to learn a complex conjunctive concept which belongs to the NO region [4]: the search remains confined in the PT region and the learned concept is over-generalized leading to a bad generalization performance.

Concerning the computational complexity, our approach is very efficient when the concept belongs to the NO region. However, it is necessary to explore more thoroughfully the search space when the problem is close to the phase transition. To speed-up our method in the YES and PT regions, we may use a simple metaalgorithm: first, to call an efficient system, like Django [13], to test if $h \theta$-subsumes $e$ then, if the answer is "false", to call our method to estimate the partial subsumption.

We plan to pursue the present study in two directions. First, we expect that the number of iterations $\mathrm{N}_{\max }$ may decrease in the PT region by coupling the two steps of our method. Second, we will implement our heuristic in some top-down and bottomup learning tools in order to verify the assumption that the gradient induced in the hypothesis space allows finding complex concepts in the NO region. 


\section{Acknowledgments}

This work is part of the ACCAMBA project (http://accamba.imag.fr) and have been funded by the ACI IMPBio. We gratefully acknowledge S. Roy and S. Aci for helpful discussions, J. Maloberti who provided his implementation of Django and the reviewers of ECML-PKDD 2006 whose comments helped to clarify this work.

\section{References}

1. Arias M., Khardon R., Maloberti J.: Learning horn expressions with LOGAN-H. Technical report 2005-4 of the Department of Computer Science, Tufts University (2005)

2. Bisson G.: Learning in FOL with a similarity measure. In Proceeding of 10th AAAI Conference, San-Jose. (1992) 82-87

3. Bisson G.: Why and how to define a similarity measure for object-based representation systems. Proceedings of 2 nd international conference on building and sharing very largescale knowledge bases (KBKS). IOS press. Enschede (NL). (1995), 236-246

4. Botta M., Saitta L., Sebag M.: Relational learning as search in a critical region. Journal of Machine Learning research, 4 (2003) 431-463.

5. Costa V. S., Srinivasan A., Camacho, R., Blockeel H., Demoen B., Janssens G., Struyf J., Vandecastaele H., Van Laer, W.: Query transformations for improving the efficiency of ilp systems. Machine Learning Research, 4 (2003) 465-491

6. Giordana A., Saitta L.: Phase transitions in relational learning. Machine Learning 41 (2000) $17-25$

7. Gottlob G., Fermüller C. G.: Removing redundancy from a clause. Artificial Intelligence, 61(2) (1993) 263-289

8. Hirata K.: On condensation of a clause. In Horvath T. and Yamamoto A., Eds, Proceedings of the 15th Int. Conf. on Inductive Logic Programming, LNAI, SpringerVerlag (2003) 164-179

9. Hogg T., Huberman B. A., Williams C. P.: Eds, Artificial Intelligence: Special Issue on Frontiers in Problem Solving: Phase Transition and Complexity, Elsevier 81 (1996) 1-2

10. Hogg T.: Refining the phase transition in combinatorial search. Artificial Intelligence, 81 (1996) 127-154

11. Khardon R.: Learning function-free horn expressions. Machine Learning, 37(3) (1999) 241-275

12. Maloberti J.: Improving Inductive Logic Programming with Constraint Satisfaction Techniques: Applications to Frequent Query Discovery. PhD thesis, LRI, Université d'Orsay (2005)

13. Maloberti J., Sebag M.: Fast theta-subsumption with constraint satisfaction algorithms. Machine Learning, 55(2) (2004) 137-174

14. Minton S., Philips A., Johnston M. D., Laird P.: Minimizing conflicts: A heuristic repair method for constraint-satisfaction and scheduling problems. Journal of Artificial Intelligence Research, 1 (1993) 1-15

15. Muggleton S., De Raedt L.: Inductive logic programming: Theory and methods. Journal of Logic Programming, 19 (1994) 629-679

16. Prosser P.: An empirical study of phase transitions in binary constraint satisfaction problems. Artificial Intelligence, 81 (1996) 81-109

17. Plotkin G.: A note on inductive generalization. Machine Intelligence, 5 (1970)

18. Smith B. M., Dyer M. E.: Locating the phase transition in binary constraint satisfaction problems. Artificial Intelligence, 81 (1996) 155-181 